\title{
ON CONNECTIONS BETWEEN THE THEORY OF RANDOM OPERATORS AND THE THEORY OF RANDOM MATRICES
}

\author{
L. PASTUR
}

To the memory of M. Sh. Birman

\begin{abstract}
For several families of selfadjoint ergodic operators, it is proved that, as the parameter that indexes the operators of a family tends to infinity, the integrated density of states converges weakly to the infinite size limit of the normalized counting measure of eigenvalues of certain random matrices. The subsequent informal discussion is devoted to the role of these results as possible indications of the presence of the continuous spectrum for random ergodic operators belonging to the families under consideration, when the indexing parameter values are sufficiently large.
\end{abstract}

\section{$\S 1$. INTRODUCTION}

In this paper, we deal with certain common topics of and links between two branches of spectral theory and mathematical physics: the random operator theory (ROT) and the random matrix theory (RMT). Both branches appeared in the 1950s and, for the most part, have been developing independently.

The RMT owes a lot to Wigner, who proposed in the early 1952 to use real symmetric and Hermitian matrices of large size with independent entries to describe the experimentally discovered phenomenon of the energy level repulsion of heavy nuclei. However, it should be mentioned that another class of large random matrices, known as sample covariance matrices, has been used in statistics since the early 1930s, although with different motivations and goals.

The ROT dates back to the seminal paper by P. Anderson of 1958, in which it was proposed to explain the absence of mobility for particles and charges in disordered media via the pure point nature of the spectrum of the corresponding finite difference and differential operators with random coefficients, namely, of the operators describing the dynamics of elementary excitations of media.

A number of other problems, results, and links with various domains of physics and mathematics have appeared since then in the ROT and the RMT, but till now the RMT is mostly concentrated on the asymptotic studies as $n \rightarrow \infty$ of eigenvalue distributions of $n \times n$ Hermitian, real symmetric, unitary, etc. random matrices for which the number $\nu_{n}$ of nonzero entries (that, as a rule, have one and the same order of magnitude) grows faster than the matrix size $n$ as $n \rightarrow \infty$ (see, e.g., [27])

On the other hand, the ROT deals mostly with eigenvectors, i.e., with spectral types of finite difference and differential operators in $\mathbb{R}^{d}$ and $\mathbb{Z}^{d}, d \geq 1$, with ergodic (and weakly dependent as a rule) coefficients, although the asymptotic properties of eigenvalues of operators defined by the same finite difference or differential expressions in large boxes are also of interest [12, 36].

2010 Mathematics Subject Classification. Primary 15B52, 60B20, 47B80.

Key words and phrases. Random matrices, random ergodic operators, integrated density of states. 
In the archetypical case of matrices with Gaussian entries (Gaussian orthogonal ensemble (GOE) and Gaussian unitary ensemble (GUE)) we have $\nu_{n}=O\left(n^{2}\right), n \rightarrow \infty$, but it can also happen that $\nu_{n} / n$ tends to infinity arbitrarily slow as $n \rightarrow \infty$, as in the case of band (see (2.3) ) or sparse random matrices. For these matrices, there is no operator in $l^{2}(\mathbb{Z})$ for which they are restrictions to finite intervals. Moreover, to prevent their spectrum from escaping to infinity as $n \rightarrow \infty$, we need to assume that the matrix entries vanish in this limit with a rate determined by $\nu_{n}$.

The simplest example of "the opposite case" is the diagonal random matrix, for which $\nu_{n}=n$. Another much less simple example is a three-diagonal or Jacobi matrix, where only the principal and the two adjacent diagonals are nonzero, so that $\nu_{n}=3 n-2$. In these cases, unlike random matrices with $\nu_{n} / n \rightarrow \infty, n \rightarrow \infty$, the nonzero entries need not vanish as $n \rightarrow \infty$, and as a rule it is assumed that the entries do not depend on $n$. An important property of these random matrices is that for them there exist "limiting objects", selfadjoint operators in $l^{2}(\mathbb{Z})$, defined by the double infinite version of the corresponding finite size matrices, i.e., by a second order finite difference equation on the entire line. These and analogous selfadjoint operators in $l^{2}\left(\mathbb{Z}^{d}\right)$ and $L^{2}\left(\mathbb{R}^{d}\right)$ defined by finite difference and differential equations with random ergodic coefficients are studied in the spectral theory of random operators (ROT) and its theoretical physics counterpart, known as the theory of disordered systems [24, 36, 12.

Our goal in this paper is to discuss certain problems and quantities that are of common interest for both the RTM and the ROT. To this end we consider several families of random ergodic operators, each family displaying certain properties pertinent to random matrices as the parameter that indexes the operators of the family tends to infinity. Part of these results dates back to [22. However, we obtain them by a simpler and more transparent method worked out recently in the RMT [34, 39, 25] and also applicable to other families. Furthermore, we use the method to analyze two more families of random operators (see (3.12)-(3.13) and (2.9) ), not considered in [22.

The organization of the paper is as follows. First, we recall the most known and studied random matrices and their properties. Then we present certain families of random ergodic operators and show that their integrated density of states (IDS) converges weakly to the limiting normalized counting measure of eigenvalues (NCM) of related random matrices. Then we give an informal discussions of implications of the results obtained, in view of recent developments of the RMT and the ROT.

\section{§2. Most Widely KNOWN RANDOM MATRICES}

2.1. Description. Here we present several most known and studied (often called classical) Hermitian random matrices and their properties related to the subject of the paper. Note that the RMT deals also with other important classes of random matrices, e.g., real symmetric, unitary, orthogonal, etc. For more detailed information on these topics, see the surveys [3, 5, 16, 35]

2.1.1. The Gaussian unitary ensemble (GUE). This is a Hermitian random matrix of size $n \times n$ defined as follows:

$$
M_{n}=n^{-1 / 2} W_{n}, \quad W_{n}=\left\{W_{j k}\right\}_{j, k=1}^{n}, \quad \overline{W_{j k}}=W_{k j} \in \mathbb{C},
$$

where the $W_{j k}, 1 \leq j \leq k \leq n$, are independent complex Gaussian random variables such that

$$
\mathbf{E}\left\{W_{j k}\right\}=\mathbf{E}\left\{W_{j k}^{2}\right\}=0, \quad \mathbf{E}\left\{\left|W_{j k}\right|^{2}\right\}=w^{2}>0
$$


Band version. This is a Hermitian random $(n \times n)$-matrix $M_{n, b}$ with the entries

$$
\begin{aligned}
\left(M_{n, b}\right)_{j k} & =b^{-1 / 2} \varphi(|j-k| / \beta) W_{j k}, \quad b=2 \beta+1, \quad \beta \in \mathbb{N}, \quad j, k=1, \ldots, n, \\
\operatorname{supp} \varphi & =[0,1], \quad \int \varphi^{2}(t) d t=1 .
\end{aligned}
$$

In particular, if in (2.1) $\varphi$ is the indicator of $(0,1)$, then $M_{n, b}$ is obtained from the GUE matrix (2.1), (2.2) by replacing by zeros the matrix elements outside the band of width $2 \beta+1$ centered around the principal diagonal.

Deformed version. In this case we have

$$
M_{n}=M_{n}^{(0)}+n^{-1 / 2} W_{n},
$$

where $M_{n}^{(0)}$ is a Hermitian $(n \times n)$-matrix, either nonrandom or random and independent of $W_{n}$.

We also mention a natural generalization of the GUE matrix, known in the RMT as the Hermitian Wigner matrix. It is a Hermitian $(n \times n)$-matrix of the same form as (2.1) but with arbitrary independent (and, possibly, $n$-dependent) $W_{j k}^{(n)}, 1 \leq j \leq k \leq n$, satisfying (2.2).

2.1.2. Hermitian Wishart matrices. These are Hermitian random $(n \times n)$-matrices of the form

$$
M_{n}=n^{-1} X_{m, n}^{*} X_{m, n}, \quad X_{m, n}=\left\{X_{\alpha j}\right\}_{\alpha, j}^{m, n},
$$

where the $\left\{X_{\alpha j}\right\}_{\alpha, j}^{m, n}$ are complex random variables such that (cf. (2.1))

$$
\mathbf{E}\left\{X_{\alpha j}\right\}=\mathbf{E}\left\{X_{\alpha j}^{2}\right\}=0, \quad \mathbf{E}\left\{\left|X_{\alpha j}\right|^{2}\right\}=a^{2}>0 .
$$

Note that in statistics the term Wishart matrices is used for those with real Gaussian random variables satisfying (2.6) (see, e.g., 30]). They are the sample covariance matrices of uncorrelated Gaussian parameters (populations). The above case of complex Gaussian random variables is known in the RMT as the Laguerre ensemble.

Deformed versions (both additive and multiplicative). In this case we have

$$
M_{n}=M_{n}^{(0)}+n^{-1} X_{m, n}^{*} T_{m} X_{m, n},
$$

where the $M_{n}^{(0)}$ and $T_{m}$ are either nonrandom or random Hermitian matrices independent of $X_{m, n}$ and of one another. Yet another version is the so-called "signal-noise" matrix

$$
M_{n}=\left(A_{m, n}+X_{m, n}\right)^{*} T_{m}\left(A_{m, n}+X_{m, n}\right),
$$

where $A_{m, n}$ is an $(n \times m)$-matrix that is either nonrandom or random but independent of $X_{m, n}$.

Replacing $\left\{X_{\alpha j}\right\}_{\alpha, j}^{m, n}$ by arbitrary independent $\left\{X_{\alpha j}^{(m, n)}\right\}_{\alpha, j}^{m, n}$ random variables with the same first two moments, we obtain sample covariance matrices of non-Gaussian populations. A similar generalization is also natural for (2.7) and (2.8).

2.1.3. Law of addition. Here we have Hermitian $(n \times n)$-matrices of the form

$$
M_{n}=A_{n}+U_{n}^{*} B_{n} U_{n},
$$

where $U_{n}$ is uniformly (Haar) distributed over $U(n)$ and $A_{n}$ and $B_{n}$ are either nonrandom or random Hermitian matrices independent of $U_{n}$ and of one another. A motivation for this problem is provided by the free probability studies (see, e.g., [4]) 
2.2. Basic results. We introduce the normalized counting measure (NCM) $N_{n}$ of the eigenvalues $\left\{\lambda_{l}^{(n)}\right\}_{l=1}^{n}$ of $M_{n}$ :

$$
N_{n}(\Delta)=\sharp\left\{l=1, \ldots, n: \lambda_{l}^{(n)} \in \Delta\right\} / n, \quad \Delta \subset \mathbb{R},
$$

known also as the empirical eigenvalue distribution. Assume that the corresponding measures $N_{n}^{(0)}$ for $M_{n}^{(0)}$ and $\sigma_{m}$ for $T_{m}$ in (2.4) and (2.7), and also $N_{A_{n}}$ for $A_{n}$ and $N_{B_{n}}$ for $B_{n}$ in (2.9) have weak limits (with probability 1 if random) as $m=m_{n} \rightarrow \infty, n \rightarrow \infty$, $m_{n} / n \rightarrow c \in[0, \infty)$. Then in all above cases $N_{n}$ converges weakly with probability 1 to a nonrandom limit $N$. This limit can be found via its Stieltjes transform

$$
f(z)=\int \frac{N(d \lambda)}{\lambda-z}, \quad \operatorname{Im} z \neq 0,
$$

which solves functional equations below, and via the inversion formula (see, e.g., [2])

$$
N(\Delta)=\lim _{\varepsilon \rightarrow 0} \frac{1}{\pi} \int_{\Delta} \operatorname{Im} f(\lambda+i 0) d \lambda .
$$

2.2.1. Deformed Gaussian unitary ensemble. (i) The Stieltjes transform of the infinite size limit with probability 1 of the $\mathrm{NCM}(2.10)$ solves the equation

$$
f(z)=f^{(0)}\left(z+w^{2} f(z)\right)
$$

where $f^{(0)}$ is the Stieltjes transform of $N^{(0)}$. This equation is uniquely solvable in the class of Nevanlinna functions, i.e., those analytic for nonreal $z$ and satisfying (see, e.g., [2])

$$
\operatorname{Im} f(z) \operatorname{Im} z \geq 0, \quad f(z)=-\frac{1}{z}+o(1 / z), \quad z \rightarrow \infty .
$$

The corresponding limiting measure is known as the deformed semicircle law.

The same result is valid for the deformed Wigner matrices (macroscopic universality) under the condition

$$
\lim _{n \rightarrow \infty} n^{-2} \sum_{1 \leq j \leq k \leq n} \mathbf{P}\left\{\left|W_{j k}\right|>\tau \sqrt{n}\right\}=0, \quad \tau>0,
$$

resembling the Lindeberg condition of probability theory [33, 25]. $N$ is absolutely continuous and has continuous density $\rho$.

In particular, if $M_{n}^{(0)}=0$ (GUE, Wigner), then from (2.11), (2.12) we obtain the semicircle law by Wigner

$$
\begin{aligned}
f(z) & =\frac{1}{2 w^{2}}\left(\sqrt{z^{2}-4 w^{2}}-z\right), \\
N(d \lambda) & =\rho(\lambda) d \lambda, \quad \rho(\lambda)=\mathbf{1}_{[-2 w, 2 w]}(\lambda) \sqrt{4 w^{2}-\lambda^{2}} .
\end{aligned}
$$

The same is true for the band matrices (2.3) with an $n$-dependent $b=b_{n}$ and $b_{n} / n \rightarrow 0$, $n \rightarrow \infty$ (see [11 for further results on the convergence of densities of measures and estimates for the error terms).

(ii) If $\lambda_{0}$ belongs to the interior (bulk) of the support of $N$ and

$$
E_{n}(s)=\mathbf{P}\left\{\left[\lambda_{0}, \lambda_{0}+s / \rho\left(\lambda_{0}\right)\right] \notin \lambda_{l}^{(n)}, l=1, \ldots, n\right\}
$$

is the gap probability, then for the deformed GUE we have the Gaudin-Wigner-Dyson law for

$$
E(s)=\lim _{n \rightarrow \infty} E_{n}(s)=\operatorname{det}(1-S(s)),
$$

where

$$
(S(s) f)(x)=\int_{0}^{s} \frac{\sin \pi(x-y)}{\pi(x-y)} f(y) d y .
$$


In particular, for the limiting probability density $p(s)=E^{\prime \prime}(s)$ of spacing between adjacent eigenvalues we have

$$
p(s)=\frac{\pi}{36} s^{2}(1+o(1)), \quad s \rightarrow 0,
$$

which demonstrates the eigenvalue repulsion phenomenon [27, 9, 20, 42].

(iii). The unitary matrix whose columns are eigenvectors of the GUE matrix is uniformly (Haar) distributed over $U(n)$, i.e., all the components of eigenvectors are typically of the same order of magnitude. This can be viewed as an analog of the nondecaying (at infinity) generalized eigenfunctions of the absolutely continuous spectrum of differential and finite difference operators (e.g., the plane waves for operators with constant coefficients), or, in physics terms, the eigenvector delocalization (see $\$ 5$ for more on this point).

Not long ago, it was shown (see, e.g., [14, 45]) that the eigenvectors of Wigner matrices are also typically delocalized, i.e., if $\psi_{l}^{(n)}=\left\{\psi_{l j}^{(n)}\right\}_{j=1}^{n},\left\|\psi_{l}^{(n)}\right\|=1$, is an eigenvector corresponding to the eigenvalue $\lambda_{l}^{(n)}$, then there exist $n$-independent $A>0$ and $C<\infty$ such that for any $K>1$ we have

$$
\mathbf{P}\left\{\max _{1 \leq j, l \leq n}\left|\psi_{l j}^{(n)}\right| \leq(\log n)^{A} / n^{1 / 2}\right\} \geq 1-C / n^{K} .
$$

2.2.2. Deformed Wishart matrices. The Stieltjes transform of the infinite size limit with probability 1 of the NCM (2.10) is a unique Nevanlinna solution of the equation

$$
f(z)=f^{(0)}\left(z-a^{2} c \int \frac{\tau \sigma(d \tau)}{1+a^{2} \tau f(z)}\right),
$$

where $c=\lim _{n \rightarrow \infty} m / n$.

The same result is valid for a wide class of sample covariance matrices with nonGaussian entries 26, 5, 25, 32

In particular, for $M_{n}^{(0)}=0$ and unit $T_{m}$ in (2.7) we have

$$
N_{M P}(d \lambda)=(1-c)_{+} \delta(\lambda) d \lambda+\rho_{M P}(\lambda) d \lambda,
$$

where (cf. (2.14) $)$

$$
\rho_{M P}(\lambda)=\left(2 \pi a^{2} \lambda\right)^{-1} \sqrt{\left(a_{+}-\lambda\right)\left(\lambda-a_{-}\right)} \mathbf{1}_{\left[a_{+}, a_{-}\right]}, \quad a_{ \pm}=a^{2}(1 \pm \sqrt{c})^{2} .
$$

Note also that for the Hermitian Wishart matrices and their non-Gaussian counterparts the properties described in items (ii) and (iii) are also fulfilled [14, 46]. This is a manifestation of the universality properties of random matrices (see, e.g., [14, 37] for recent results).

2.2.3. Law of addition. Here also the limiting NCM of (2.9) can be found via its Stieltjes transform, which satisfies the following system of functional equations determined by the Stieltjes transforms $f_{A}$ and $f_{B}$ of the limiting NCM of $\left\{A_{n}\right\}$ and $\left\{B_{n}\right\}$ :

$$
\begin{aligned}
f(z) & =f_{A}\left(h_{B}(z)\right), \\
f(z) & =f_{B}\left(h_{A}(z)\right), \\
f^{-1}(z) & =z-h_{A}(z)-h_{B}(z) .
\end{aligned}
$$

This system is uniquely solvable in the class of functions $\left(f, h_{A}, h_{B}\right)$ analytic for nonreal $z$, satisfying (2.12), and such that

$$
h_{A, B}(z)=z+O(1), \quad z \rightarrow \infty ;
$$

see [38, 39]. 


\section{§3. FAMILIES OF RANDOM OPERATORS}

Here we describe the families of ergodic operators that we are going to study. The random parts of the operators resemble those of random matrices of $\S 2$. We recall also the required properties of the integrated density of states for these operators.

3.1. Description. (i). Define a symmetric random operator $H_{R_{G}}$ in $l_{2}\left(\mathbb{Z}^{d}\right), d \geq 1$, by indicating its matrix $\left\{H_{R_{G}}(x, y)\right\}_{x, y \in \mathbb{Z}^{d}}$ as follows

$$
H_{R_{G}}(x, y)=h(x-y)+R_{G}^{-d / 2} \varphi\left((x-y) / R_{G}\right) W(x, y), \quad x, y \in \mathbb{Z}^{d},
$$

where $h: \mathbb{Z}^{d} \rightarrow \mathbb{C}$,

$$
h(-x)=\overline{h(x)}, \quad \sum_{x \in \mathbb{Z}^{d}}|h(x)|^{2}<\infty
$$

$R_{G}>0, \varphi: \mathbb{R}^{d} \rightarrow \mathbb{R}$ is piecewise continuous,

$$
\max _{t \in \mathbb{R}_{\mathbb{G}}}|\varphi(t)| \leq \varphi_{0}<\infty, \quad \varphi(t)=0,|t|>1, \quad \int_{\mathbb{R}^{d}} \varphi^{2}(t) d t=1,
$$

and (cf. (2.2)

$$
W(x, y)=\overline{W(y, x)}, \quad x, y \in \mathbb{Z}^{d},
$$

are independent (modulo the above symmetry condition) complex Gaussian random variables such that (cf. (2.2) )

$$
\begin{gathered}
\mathbf{E}\{W(x, y)\}=\mathbf{E}\left\{W(x, y)^{2}\right\}=0, \\
\mathbf{E}\left\{|W(x, y)|^{2}\right\}=w^{2}>0, \quad x, y \in \mathbb{Z}^{d} .
\end{gathered}
$$

$\delta$ being the delta-symbol.

In the case of $d=1$, the random part of $H_{R_{G}}$ of (3.1) is an infinite matrix having nonzero entries only inside the band of width $\left(2 R_{G}+1\right)$ around the principal diagonal; this matrix can be viewed as an infinite-dimensional analog of the band matrix (2.3).

(ii) Define a symmetric random operator $H_{d}=\left\{H_{d}(x, y)\right\}_{x, y \in \mathbb{Z}^{d}}$ in $l_{2}\left(\mathbb{Z}^{d}\right)$ by

$$
H_{d}(x, y)=h_{d}(x-y)+(2 d)^{-1 / 2} W_{1}(x, y),
$$

where, for $x=\left(x_{1}, \ldots, x_{d}\right), \delta$ is the delta-symbol,

$$
h_{d}(x)=d^{-1 / 2} \sum_{j=1}^{d} h_{1}\left(x_{j}\right) \prod_{k \neq i} \delta\left(x_{k}\right), \quad h(0)=0,
$$

$h_{1}: \mathbb{Z} \rightarrow \mathbb{C}$ satisfies (3.2) for $d=1$ (e.g., $h_{d}$ may be the matrix of the discrete Laplacian), and

$$
W_{1}(x, y)= \begin{cases}W(x, y), & |x-y|=1, \\ 0, & |x-y| \neq 1,\end{cases}
$$

and the $W(x, y)$ are as in (3.5).

(iii) Define a symmetric operator

$$
H_{n_{W}}=\left\{H_{n_{W}}(x, \alpha ; y, \beta)\right\}_{x, y \in \mathbb{Z}^{d}, \alpha, \beta=1, \ldots, n_{W}}
$$

in $l_{2}\left(\mathbb{Z}^{d}\right) \otimes \mathbb{C}^{n_{W}}$ as

$$
H_{n_{W}}(x, \alpha ; y, \beta)=h(x-y) \delta_{\alpha \beta}+n_{W}^{-1 / 2} \delta(x-y) W_{\alpha \beta}(x),
$$

where $x, y \in \mathbb{Z}^{d}, \alpha, \beta=1, \ldots, n_{W}, h$ is the same as in $H_{R_{G}}$, and

$$
W_{\alpha \beta}(x)=\overline{W_{\beta \alpha}(x)}, \quad x \in \mathbb{Z}^{d}, \quad \alpha, \beta=1, \ldots, n_{W},
$$


are independent (modulo the symmetry condition) complex Gaussian random variables (cf. (2.2) and (3.5)):

$$
\begin{aligned}
\mathbf{E}\left\{W_{\alpha \beta}(x)\right\} & =\mathbf{E}\left\{W_{\alpha \beta}^{2}(x)\right\}=0, \\
\mathbf{E}\left\{\left|W_{\alpha \beta}(x)\right|^{2}\right\} & =w^{2}>0, \quad x \in \mathbb{Z}^{d}, \alpha, \beta=1, \ldots, n_{W} .
\end{aligned}
$$

The operator $H_{n_{W}}$ is a special case of those introduced by Wegner in [50. It can be regarded as the $n_{W}$-component analog of the discrete Schrödinger operator (the Anderson model (5.1) ) or as the Hamiltonian of a disordered system in the dimension $d+n_{W}$ in which the random potential in $n_{W}$ "transverse" dimensions is written in the "mean field" form.

(iv). The random part of the operator $H_{R_{G}}$ of (3.1) is the infinite random matrix

$$
\left\{R_{G}^{-d / 2} \varphi\left((x-y) / R_{G}\right) W(x, y)\right\}_{x, y \in \mathbb{Z}^{d}}
$$

resembling the GUE matrix (2.1) - (2.2) or rather the band matrix (2.3). Recalling that another random matrix constructed from complex Gaussian random variables is the Hermitian Wishart matrix (2.5), (2.6) and its deformations (2.7), (2.8), we can introduce an analog of $H_{R_{G}}$ with the Hermitian Wishart type random part:

$$
H_{R_{W}}(x, y)=h(x-y)+R_{W}^{-d} \varphi\left((x-y) / R_{W}\right) \sum_{\alpha=1}^{m} \overline{X_{\alpha}(x)} X_{\alpha}(y), \quad x, y \in \mathbb{Z}^{d},
$$

where $h$ is as in (3.1), $\left\{X_{\alpha}(x)\right\}_{1 \leq \alpha \leq m, x \in \mathbb{Z}^{d}}$ are independent and identically distributed complex Gaussian random variables such that (cf. (2.6) )

$$
\mathbf{E}\left\{X_{\alpha}(x)\right\}=\mathbf{E}\left\{X_{\alpha}^{2}(x)\right\}=0, \quad \mathbf{E}\left\{\left|X_{\alpha}(x)\right|^{2}\right\}=a^{2}>0,
$$

and $\varphi$ is a positive definite function vanishing sufficiently fast at infinity.

(v) The random part of the next ergodic operator is due to unitary Haar distributed random matrices, cf. (2.9). Namely, consider the symmetric operator $H_{n_{V}}$ in $l^{2}\left(\mathbb{Z}^{d}\right) \otimes \mathbb{C}^{n_{V}}$ defined by the matrix (cf. (3.9)-(3.11)

$$
H_{n_{V}}(x, \alpha ; y, \beta)=h(x-y) \delta_{\alpha \beta}+\delta(x-y)\left(U_{n_{V}}^{*}(x) B_{n_{V}} U_{n_{V}}(x)\right)_{\alpha \beta},
$$

where $h$ is as above, $x, y \in \mathbb{Z}^{d}, \alpha, \beta=1, \ldots, n_{V},\left\{U_{n_{V}}(x)\right\}_{x \in \mathbb{Z}^{d}}$ are independent and identically distributed unitary $\left(n_{V} \times n_{V}\right)$-matrices whose common probability law is the normalized Haar measure on $U\left(n_{V}\right)$, and $B_{n_{V}}$ is a Hermitian $\left(n_{V} \times n_{V}\right)$-matrix.

The random operators $H_{R_{G}}, H_{R_{W}}$, and $H_{d}$ can be viewed as analogs of the Hamiltonians of lattice models of statistical mechanics in which $R_{G}$ and $R_{W}$ are analogs of the interaction radius and $d$ is the dimension of the space. The limits $R_{G, W} \rightarrow \infty$ and $d \rightarrow \infty$ in these Hamiltonians lead to the mean field models which, being rather simple, provide nevertheless a fairly reasonable qualitative description of the corresponding systems with large interaction radius and in high dimensions (see [18, 21]). Similarly, the operators $H_{n_{W}}$ and $H_{n_{V}}$ are analogs of the Hamiltonians of statistical mechanics models having a certain internal structure (a fixed number spin components or orbitals per site (see [21, 44, 50])), and here the limit of the infinite number of spin components or orbitals leads to the spherical model.

Note also that the five families (3.1)-(3.14) of random operators have the form of the sum of a nonrandom translation invariant part and a fluctuating random part explicitly involving the parameters $R_{G}, d, R_{W}, n_{W}, n_{V}$ that we are going to send to infinity. The random parts are such that the larger these parameters are, the more "extended" and smaller the randomness is. This again resembles the statistical mechanics setup, where a similar scaling of the interaction is widely used in the mean field and the spherical approximations. 
3.2. Integrated density of states. We denote by $\Omega$ the infinite-dimensional probability space formed by $\{W(x . y)\}_{x, y \in \mathbb{Z}^{d}}$ for $a=R_{G}, d$, i.e., in the case of (3.4) and (3.5); by $\left\{W_{\alpha \beta}(x, y)\right\}_{\alpha, \beta=1, \ldots, n ; x, y \in \mathbb{Z}^{d}}$ for $a=n_{W}$, i.e., in the case of (3.10) and (3.11); by $\left\{X_{\alpha}(x)\right\}_{\alpha \in \mathbb{N} ; x \in \mathbb{Z}^{d}}$ for $a=R_{W}$, i.e., in the case of (3.12); and by $\left\{U_{n}(x)\right\}_{x \in \mathbb{Z}^{d}}$ for $a=n_{V}$. Let $\left\{T_{s}\right\}_{s \in \mathbb{Z}^{d}}$ be the (shift) automorphisms of $\Omega$ defined by

$$
W\left(x, y, T_{s} \omega\right)=W(x+s, y+s, \omega), \quad x, y \in \mathbb{Z}^{d},
$$

for $a=R_{G}, d$, by

$$
W_{\alpha \beta}\left(x, y, T_{s} \omega\right)=W_{\alpha \beta}(x+s, y+s, \omega), \quad x, y \in \mathbb{Z}^{d}, \quad \alpha, \beta=1, \ldots, n_{W},
$$

for $a=n_{W}$,

for $a=R_{W}$, and by

$$
X_{\alpha}\left(x, T_{s} \omega\right)=X_{a}(x+s), \quad \alpha=1, \ldots, m,
$$

for $a=n_{V}$.

The definitions of the corresponding collections of random variables show that each $T_{s}$ preserves the probability measure in $\Omega$ and that $\left\{T_{s}\right\}_{s \in \mathbb{Z}^{d}}$ is an ergodic group of automorphisms of $\Omega$. This implies that the random operators $H_{a}, a=R_{G}, d, R_{W}, n_{W}, n_{V}$ are symmetric ergodic operators in the sense of [36, $\S \S 1 . \mathrm{D}$ and 2.A]. Furthermore, let $\left\{U_{s}\right\}_{s \in \mathbb{Z}^{d}}$ be the group of unitary (shift) operators in $l^{2}\left(\mathbb{Z}^{d}\right)$ defined for $\psi \in l^{2}\left(\mathbb{Z}^{d}\right)$ by

$$
\left(U_{s} \psi\right)(x)=\psi(x+s), \quad x \in \mathbb{Z}^{d},
$$

if $a=R_{G}, d, R_{W}$, and defined on $l^{2}\left(\mathbb{Z}^{d}\right) \otimes \mathbb{C}^{a}$ by

$$
\left(U_{s} \psi\right)(x, \alpha)=\psi(x+s, \alpha), \quad x \in \mathbb{Z}^{d}, \quad \alpha=1, \ldots, a,
$$

if $a=n_{W}, n_{V}$. Then with probability 1 we have

$$
U_{s} H_{a}(\omega) U_{s}^{*}=H\left(T_{s} \omega\right), \quad s \in \mathbb{Z}^{d} .
$$

We refer the reader to [36, Chapters I and II] for the general spectral properties of ergodic operators. In particular, from Corollary 4.3 of [36] it follows that the symmetric operators $H_{a}$ are defined with probability 1 on the set of sequences in $x \in \mathbb{Z}^{d}$ with finite support and are essentially selfadjoint on this set.

Our intention is to study the simplest, although rather important from several points of view, spectral characteristic of the above ergodic operators, known as the integrated density of states (IDS). It is an analog of the limiting NCM of random matrices and is defined as follows. For each of the above operators, consider its "finite box" version, i.e., the restrictions $H_{a \Lambda}$ of $H_{a}$ to the cube $\Lambda \subset \mathbb{Z}^{d}$ centered at the origin. We obtain $|\Lambda| \times|\Lambda|$ Hermitian random matrices for $a=R_{G}, d, R_{W}$, and $|\Lambda| a \times|\Lambda| a$ matrices for $a=$ $n_{W}, n_{V}$. For each of them, in the usual way, we define the NCM $N_{a \Lambda}$ (see (2.10) ) of their eigenvalues as the eigenvalue counting measure divided by the size of the corresponding matrix. The general results of spectral theory of ergodic operators (see [36, Chapter IV]) show that, for each of the above operators, the random measures $N_{a \Lambda}$ converge weakly with probability 1 to a nonrandom limit $N_{a}$ as $\Lambda$ tends to $\mathbb{Z}^{d}$ (or $\mathbb{R}^{d}$ ) and that for any $\Delta \subset \mathbb{R}$ we have

$$
N_{a}(\Delta)=\mathbf{E}\left\{\mathcal{E}_{a}(0,0 ; \Delta)\right\}, \quad a=R_{G}, d, R_{W},
$$

where $\left\{\mathcal{E}_{a}(x, y ; \Delta)\right\}_{x, y \in \mathbb{Z}^{d}}$ is the matrix of the resolution of identity of $H_{a}$ for $a=$ $R_{G}, d, R_{W}$, and

$$
N_{a}(\Delta)=\mathbf{E}\left\{a^{-1} \sum_{\alpha=1}^{a} \mathcal{E}_{a}(\alpha, 0 ; \alpha, 0 ; \Delta)\right\}, \quad a=n_{W}, n_{V},
$$


where

$$
\left\{\mathcal{E}_{a}(x, \alpha ; y, \beta ; \Delta)\right\}_{x, y \in \mathbb{Z}^{d}, \alpha, \beta=1, \ldots, a}
$$

is the matrix of the resolution of identity of the operators $H_{a}$ for $a=n_{W}, n_{V}$.

\section{§4. The Limiting Integrated Density of STATES}

In this section we show that the IDS of $H_{a}, a=R_{G}, d, R_{W}, n_{W}, n_{V}$, all tend weakly as $a \rightarrow \infty$ to the limits coinciding with the infinite size limits of the normalized counting measure of the random matrix in $\S 2$ (the deformed semicircle law for $a=R_{G}, d$, and $n_{W}$, the measure defined by (2.20) for the matrices (2.7), and the law of addition of random matrices (2.9) for $a=n_{V}$ ). Moreover, the limits are closely related to certain approximations for the integrated density of states of elementary excitations in certain models of disordered condensed media (see [21, 22, 24, and the next section).

The natural condition that we should obtain a finite nonzero IDS in these limits fixes the normalization factors $R_{G}^{-1 / 2}, d^{-1 / 2}$ and $n_{W}{ }^{-1 / 2}$ in (3.1)-(3.11). This will be seen below in the full extent. Here we give a simple argument. Indeed, by (3.11) and the spectral theorem, for $a=R_{G}, d, R_{W}$ we have

$$
\int \lambda^{2} N_{a}(d \lambda)=\mathbf{E}\left\{\left(H_{a}^{2}\right)(0,0)\right\}=\sum_{x \in \mathbb{Z}^{d}} \mathbf{E}\left\{\left|H_{a}(0, x)\right|^{2}\right\},
$$

and for $a=n_{W}, n_{V}$ we have

$$
\int \lambda^{2} N_{a}(d \lambda)=a^{-1} \sum_{\alpha, \beta=1}^{a} \sum_{x \in \mathbb{Z}^{d}} \mathbf{E}\left\{H_{a}^{2}(\alpha, 0 ; \beta, x)\right\} .
$$

Then, from (3.1)-(3.5) for $a=R_{G}$ we get

$$
\sum_{x \in \mathbb{Z}^{d}} \mathbf{E}\left\{\left|H_{R_{G}}(0, x)\right|^{2}\right\}=\sum_{x \in \mathbb{Z}^{d}}|h(x)|^{2}+w^{2} R^{-d} \sum_{x \in \mathbb{Z}^{d}}\left|\varphi\left(x / R_{G}\right)\right|^{2},
$$

and conditions (3.2)-(3.3) imply that the right-hand side of this expression tends to a nonzero and finite limit:

$$
\lim _{R_{G} \rightarrow \infty} \sum_{x \in \mathbb{Z}^{d}} \mathbf{E}\left\{\left|H_{R_{G}}(0, x)\right|^{2}\right\}=\sum_{x \in \mathbb{Z}^{d}}|h(x)|^{2}+w^{2}<\infty .
$$

A similar argument applies to the other cases.

Denote by $N^{(0)}$ the IDS of the nonrandom (unperturbed) parts of the operators (3.1)(3.12). For these convolution operators, which satisfy (3.15) for $\Omega=\{0\}$, formula (3.16) implies that the relation

$$
N^{(0)}(d \lambda)=\operatorname{meas}\left\{k \in \mathbb{T}^{d}: \widehat{h}(k) \in d \lambda\right\},
$$

where $\mathbb{T}^{d}=[0,1]^{d}$ is the $d$-dimensional torus, and

$$
\widehat{h}(k)=\sum_{x \in \mathbb{Z}^{d}} h(x) e^{2 \pi i(k, x)}
$$

is the symbol of the corresponding operator.

Note that for the operator $H_{d}$ of (3.6) the nonrandom part and its IDS depends also on $d$. Therefore, unlike $H_{R_{G}}, H_{n_{W}}, H_{R_{W}}$, and $H_{n_{V}}$, in the case of $H_{d}$ the limit as $d \rightarrow \infty$ affects also the IDS of the "unperturbed" convolution operator defined by the first term on the right-hand side (3.6) and by (3.7). More precisely, in this case $N^{(0)}$ is given by the limit of (3.7) and (4.3) as $d \rightarrow \infty$ and it is the Gaussian measure

$$
N^{(0)}(d \lambda)=\left(2 \pi h_{2}\right)^{-1 / 2} e^{-\lambda^{2} / 2 h_{2}} d \lambda,
$$


where

$$
h_{2}=\sum_{x \in \mathbb{Z}} h_{1}^{2}(x) .
$$

Now we formulate the main result of the paper.

Theorem 4.1. Let $H_{a}, a=R_{G}, d, R_{W}, n_{W}, n_{V}$, be the random operators defined by (3.1)-(3.14), let $N^{(0)}$ be defined by (4.3) for $a=R_{G}, n_{W}$, and by (4.5) for $a=d$, and let $N_{a}$ be the integrated density of states of $H_{a}$ given by (3.16), (3.17). Then $N_{a}$ converges weakly as $a \rightarrow \infty$ to the probability measure $N_{\lim }$ whose Stieltjes transform $f$ solves the equations below.

(i) For $a=R_{G}, d, n_{W}$, equation (2.11) in which $f^{(0)}$ is the Stieltjes transform of (4.3) for $a=R_{G}, n_{W}$ and of (4.5) for $a=d$.

(ii) For $a=R_{W}$, equation (2.20) in which $f^{(0)}$ is the Stieltjes transform of (4.3) and

$$
\sigma(\Delta)=\text { meas }\{k \in \operatorname{supp} \widehat{\varphi}: \widehat{\varphi}(k) \in \Delta\},
$$

where $\widehat{\varphi}$ is the Fourier transform of a positive definite function $\varphi$ with compact support;

(iii) For $a=n_{V}$, equations (2.23) in which $f_{A}$ is the Stieltjes transform of (4.3) and $f_{B}$ is the limiting normalized counting measure of $\left\{N_{B_{n_{V}}}\right\}$ as $n_{V} \rightarrow \infty$ and it is assumed that

$$
\sup _{n_{V}} \int_{\mathbb{R}} \lambda^{4} N_{n_{V}}(d \lambda)<\infty .
$$

Below, we give a detailed proof for the case where $a=R_{G}$, because it is the simplest technically, but contains, however, all basic moments of the proofs for the other cases, except for certain moments of the proof for $a=n_{V}$, which is also outlined below. All the proofs follow essentially those on the limiting normalized counting measure for the corresponding random matrices, as described in \$2 (see, e.g., [34, 39]).

Lemma 4.2. Let

$$
G(z)=\left(H_{R_{G}}-z\right)^{-1}=\{G(x, y ; z)\}_{x, y \in \mathbb{Z}^{d}}
$$

be the resolvent of the operator $H_{R_{G}}$, and let

$$
G^{(0)}(z)=\left(H^{(0)}-z\right)^{-1}=\left\{G^{(0)}(x, y ; z)\right\}_{x, y \in \mathbb{Z}^{d}}
$$

be the resolvent of the nonrandom part of $H_{R_{G}}$ :

$$
H^{(0)}=\{h(x-y)\}_{x, y \in \mathbb{Z}^{d}} .
$$

Then for any nonreal $z$ we have

$$
\mathbf{E}\{G(z)\}=G^{(0)}\left(\widetilde{z}_{R_{G}}(z)\right)+\mathbf{E}\{G(z) D(z)\} G^{(0)}(\widetilde{z}(z)),
$$

where $D(z)=\{D(x, y ; z)\}_{x, y \in \mathbb{Z}^{d}}$ is the diagonal operator defined by

$$
D(x, y ; z)=\delta(x-y) D(x ; z)
$$

with

$$
D(x ; z)=R_{G}^{-d} \sum_{x^{\prime} \in \mathbb{Z}^{d}} \varphi^{2}\left(\left(x-x^{\prime}\right) / R_{G}\right)\left(G\left(x^{\prime}, x^{\prime} ; z\right)-\mathbf{E}\{G(0,0 ; z)\}\right),
$$

and

$$
\widetilde{z}_{R_{G}}(z)=z+w^{2} \mathbf{E}\{G(0,0 ; z)\} S_{R_{G}}
$$

with

$$
S_{R_{G}}=R_{G}^{-d} \sum_{x \in \mathbb{Z}^{d}}\left|\varphi\left(x / R_{G}\right)\right|^{2} .
$$


Proof. By using the resolvent identity for the pair $\left(H_{R_{G}}, H^{(0)}\right)$, we obtain

$$
\begin{aligned}
& \mathbf{E}\{G(x, y ; z)\}=G^{(0)}(x, y ; z) \\
& \quad-\mathbf{E}\left\{R_{G}^{-d / 2} \sum_{x^{\prime}, x^{\prime \prime} \in \mathbb{Z}^{d}} G\left(x, x^{\prime} ; z\right) \varphi\left(\left(x^{\prime}-x^{\prime \prime}\right) / R_{G}\right) W\left(x^{\prime}, x^{\prime \prime}\right)\right\} G^{(0)}\left(x^{\prime \prime}, y ; z\right) .
\end{aligned}
$$

Now we use the formula

$$
\left.\mathbf{E}\{\zeta \Phi(\zeta, \bar{\zeta})\}=w^{2} \mathbf{E}\left\{\frac{\partial}{\partial \bar{\zeta}} \Phi(\zeta, \bar{\zeta}\}\right)\right\}
$$

where $\Phi: \mathbb{C}^{2} \rightarrow \mathbb{C}$ is a differentiable function with bounded derivatives and $\zeta$ is the complex Gaussian variable specified by (cf. (2.2))

$$
\mathbf{E}\{\zeta\}=\mathbf{E}\left\{\zeta^{2}\right\}=0, \quad \mathbf{E}\left\{|\zeta|^{2}\right\}=w^{2} .
$$

Formula (4.17) is an abbreviated form of the relation

$$
\frac{1}{2 \pi \sigma^{2}} \int_{\mathbb{C}} z \Phi(z, \bar{z}) e^{-|z|^{2} / w^{2}} L(d z)=w^{2} \frac{1}{2 \pi \sigma^{2}} \int_{\mathbb{C}} \frac{\partial}{\partial \bar{z}} \Phi(z, \bar{z}) e^{-|z|^{2} / 2 \sigma^{2}} L(d z)
$$

(where $L(d z)$ is the Lebesgue measure in $\mathbb{C}$ ), resulting from integration by parts.

By using this formula with $G\left(x, x^{\prime} ; z\right)$ as $\Phi$ and $W\left(x^{\prime}, x^{\prime \prime}\right)$ as $\zeta$, we obtain

$$
\mathbf{E}\left\{G\left(x, x^{\prime} ; z\right) W\left(x^{\prime}, x^{\prime \prime}\right)\right\}=w^{2} \mathbf{E}\left\{\frac{\partial G\left(x, x^{\prime} ; z\right)}{\partial W\left(x^{\prime \prime}, x^{\prime}\right)}\right\} .
$$

Combining this and the formula

$$
\frac{\partial G\left(x, x^{\prime} ; z\right)}{\partial W\left(x^{\prime \prime}, x^{\prime}\right)}=-R_{G}^{-d / 2} \varphi\left(\left(x^{\prime}-x^{\prime \prime}\right) / R_{G}\right) G\left(x, x^{\prime \prime} ; z\right) G\left(x^{\prime}, x^{\prime} ; z\right),
$$

which follows from (3.1) and the resolvent identity, and using (4.13), (4.14), (4.16), we get

$$
\mathbf{E}\{G(z)\}=G^{(0)}(z)+w^{2} S_{R_{G}} \mathbf{E}\{G(0,0 ; z)\} \mathbf{E}\{G(z)\} G^{(0)}(z)+\mathbf{E}\{G(z) D(z)\} G^{(0)}(z),
$$

or

$$
\mathbf{E}\{G(z)\}\left(1-w^{2} \mathbf{E}\{G(0,0 ; z)\} S_{R_{G}} G^{(0)}(z)\right)=G^{(0)}(z)+w^{2} \mathbf{E}\{G(z) D(z)\} G^{(0)}(z) .
$$

From (4.10) and (4.15) it follows that

$$
1-w^{2} S_{R_{G}} \mathbf{E}\{G(0,0 ; z)\} G^{(0)}(z)=\left(H^{(0)}-\widetilde{z}_{R_{G}}(z)\right) G^{(0)}(z),
$$

or

$$
\mathbf{E}\{G(z)\}\left(H^{(0)}-\widetilde{z}_{R_{G}}(z)\right)=1+\mathbf{E}\{G(z) D(z)\},
$$

where $\widetilde{z}_{R_{G}}$ is defined in (4.15). Also, the inequality $\operatorname{Im} G(x, x ; z) \operatorname{Im} z>0, \operatorname{Im} z \neq 0$, valid for the resolvent of any selfadjoint operator, implies the bound $\left|\operatorname{Im} \widetilde{z}_{R_{G}}(z) \geq\right| \operatorname{Im} z \mid>0$, and thus, the invertibility of $H^{(0)}-\widetilde{z}_{R_{G}}(z)$ for $\operatorname{Im} z \neq 0$. Hence, we can multiply both sides of the last identity by $G^{(0)}\left(\widetilde{z}_{R_{G}}(z)\right)$ and obtain (4.12).

Proof of Theorem 4.1. (i) The case where $a=R_{G}$. It suffices to prove that the Stieltjes transform $f_{R_{G}}$ of $N_{R_{G}}$ converges to the solution of (2.11) uniformly on any compact set in $\mathbb{C} \backslash \mathbb{R}$. Indeed, the correspondence of the probability measures and their Stieltjes transforms is one-to-one and continuous with respect to the uniform convergence of Stieltjes transforms on compact sets in $\mathbb{C} \backslash \mathbb{R}$ and the weak convergence of probability measures, provided that the corresponding collection of measures is tight [2]. The last property follows from relations (4.1) $-(4.2)$, ensuring tail bounds for $N_{R_{G}}$ that are uniform in $R_{G}$. 
Formula (3.16) and the spectral theorem show that

$$
f_{R_{G}}(z)=\mathbf{E}\{G(0,0 ; z)\} .
$$

This and (4.12) lead to the relation

$$
f_{R_{G}}(z)=f^{(0)}\left(z+w^{2} f_{R_{G}}(z)\right)+\varepsilon_{R_{G}}(z),
$$

where

$$
\varepsilon_{R_{G}}(z)=\mathbf{E}\left\{\left(G(z) D(z) G^{(0)}\left(\widetilde{z}_{R_{G}}(z)\right)\right)(0,0)\right\}
$$

and $D(z)$ is given by (4.13)-4.14). The Schwarz inequality yields

$$
\begin{aligned}
\left|\varepsilon_{R_{G}}(z)\right|^{2} & \leq\left|\sum_{x \in \mathbb{Z}^{d}} \mathbf{E}\{G(0, x ; z) D(x ; z)\} G^{(0)}(x, 0 ; z)\right|^{2} \\
& \leq \sum_{x \in \mathbb{Z}^{d}} \mathbf{E}\left\{|G(0, x ; z)|^{2}\right\} \sum_{x \in \mathbb{Z}^{d}} \mathbf{E}\left\{|D(x ; z)|^{2}\right\}\left|G^{(0)}\left(x, 0 ; \widetilde{z}_{R_{G}}\right)\right|^{2} .
\end{aligned}
$$

Now, using (4.13), (4.14) and the Schwarz inequality once again, we obtain

$$
\mathbf{E}\left\{|D(x ; z)|^{2}\right\} \leq S_{R_{G}} \sum_{x^{\prime} \in \mathbb{Z}^{d}} R_{G}^{-d} \varphi^{2}\left(\left(x-x^{\prime}\right) / R_{G}\right) \mathbf{E}\left\{\left|G\left(x^{\prime}, x^{\prime} ; z\right)-f_{R_{G}}(z)\right|^{2}\right\} .
$$

By (3.15) and (4.21), we can write

$$
\mathbf{E}\left\{G\left(x^{\prime}, x^{\prime} ; z\right)\right\}=f_{R_{G}}(z), \quad \mathbf{E}\left\{\left|G\left(x^{\prime}, x^{\prime} ; z\right)\right|^{2}\right\}=\mathbf{E}\left\{|G(0,0 ; z)|^{2}\right\}, \quad x^{\prime} \in \mathbb{Z}^{d},
$$

whence

$$
\mathbf{E}\left\{\left|G\left(x^{\prime}, x^{\prime} ; z\right)-f_{R_{G}}(z)\right|^{2}\right\}=\operatorname{Var}\{G(0,0 ; z)\} .
$$

Now we use the following bound for the variance. Let $\left\{\xi_{\alpha}\right\}_{\alpha \in A}$ be a collection of independent real Gaussian random variables satisfying

$$
\mathbf{E}\left\{\xi_{\alpha}\right\}=0, \quad \mathbf{E}\left\{\xi_{\alpha}^{2}\right\}=w^{2}
$$

(cf. (2.2) for $j=k$ and (3.5) for $x=y$ ), and let $\left\{\zeta_{\beta}\right\}_{\beta \in B}$ be a collection of independent complex Gaussian random variables satisfying

$$
\mathbf{E}\left\{\zeta_{\beta}\right\}=\mathbf{E}\left\{\zeta_{\beta}^{2}\right\}=0, \quad \mathbf{E}\left\{\left|\zeta_{\beta}\right|^{2}\right\}=w^{2}
$$

(cf. (2.2) for $j \neq k$ and (3.5) for $x \neq y$ ). If $\Phi$ is a complex-valued function of the variables $\left\{x_{\alpha}\right\}_{\alpha \in A}$ and $\left\{z_{\beta}, \bar{z}_{\beta}\right\}_{\beta \in B}$ and $\Phi$ has bounded partial derivatives with respect to all variables, then for the random variable

$$
\widehat{\Phi}=\left.\Phi\right|_{x_{a}=\xi_{a}, \alpha \in A ; z_{\beta}=\zeta_{\beta}, \bar{z}_{\beta}=\bar{\zeta}_{\beta}, \beta \in B}
$$

obtained by replacing the variables $\left\{x_{\alpha}\right\}_{\alpha \in A}$ in $\Phi$ by the random variables $\left\{\xi_{\alpha}\right\}_{\alpha \in A}$ and $\left\{z_{\beta}, \bar{z}_{\beta}\right\}_{\beta \in B}$ by $\left\{\zeta_{\beta}, \bar{\zeta}_{\beta}\right\}_{\beta \in B}$, we have

$$
\begin{aligned}
\operatorname{Var}\{\widehat{\Phi}\} & :=\mathbf{E}\left\{|\widehat{\Phi}|^{2}\right\}-|\mathbf{E}\{\widehat{\Phi}\}|^{2} \\
& \leq w^{2} \sum_{\alpha \in A} \mathbf{E}\left\{\left|\widehat{\Phi}_{x_{a}}\right|^{2}\right\}+\frac{w^{2}}{2} \sum_{\beta \in B} \mathbf{E}\left\{\left|\widehat{\Phi}_{z_{\beta}}\right|^{2}+\left|\widehat{\Phi}_{\bar{z}_{\beta}}\right|^{2}\right\},
\end{aligned}
$$

where $\widehat{\Phi}_{x_{a}}$ is the random variable obtained by replacing the variables $\left\{x_{\alpha}\right\}_{\alpha \in A}$ in $\frac{\partial \Phi}{\partial x_{\alpha}}$ by the random variables $\left\{\xi_{\alpha}\right\}_{\alpha \in A}$ and $\left\{z_{\beta}, \bar{z}_{\beta}\right\}_{\beta \in B}$ by $\left\{\zeta_{\beta}, \bar{\zeta}_{\beta}\right\}_{\beta \in B}$, and $\widehat{\Phi}_{z_{\beta}}$ and $\widehat{\Phi}_{\bar{z}_{\beta}}$ are obtained from $\frac{\partial \Phi}{\partial z_{\beta}}$ and $\frac{\partial \Phi}{\partial \bar{z}_{\beta}}$ by the same substitution.

The above bound is a version of the Poincaré-Nash inequality for products of Gaussian measures (see, e.g., [8]).

By using this bound for $G(0,0 ; z)$ in the role of $\widehat{\Phi},\{W(x, x)\}_{x \in \mathbb{Z}^{d}}$ in the role of $\left\{\xi_{\alpha}\right\}_{\alpha \in A}$, and $\{W(x, y)\}_{(x, y) \in \mathbb{Z}_{s y m}^{2 d}}$ (where $\mathbb{Z}_{\text {sym }}^{2 d}$ is $\mathbb{Z}^{d} \times \mathbb{Z}^{d}$ in which $(x, y)$ and $(y, x)$ are 
identified) in the role of $\left\{\zeta_{\beta}\right\}_{\beta \in B}$, and taking (4.20) into account, after little algebra we obtain the inequality

$$
\begin{aligned}
& \mathbf{E}\left\{\left|G(0,0 ; z)-f_{R_{G}}(z)\right|^{2}\right\} \\
& \quad \leq w^{2} \sum_{x_{1}, x_{2} \in \mathbb{Z}^{d}} R_{G}^{-d}\left|\varphi\left(\left(x_{1}-x_{2}\right) / R_{G}\right)\right|^{2} \mathbf{E}\left\{\left|G\left(0, x_{1} ; z\right)\right|^{2}\left|G\left(0, x_{2} ; z\right)\right|^{2}\right\} \\
& \quad \leq \varphi_{0}^{2} \mathbf{E}\left\{|G(z) G(\bar{z})(0,0)|^{2}\right\} / R_{G}^{d} \leq \varphi_{0}^{2} / R_{G}^{d}|\operatorname{Im} z|^{4} .
\end{aligned}
$$

Here we have used (3.3), the relation

$$
\sum_{x \in \mathbb{Z}^{d}}\left|G\left(0, x_{1} ; z\right)\right|^{2}=\left(G G^{*}\right)(0,0 ; z),
$$

and the bounds

$$
\left.\left|\left(G G^{*}\right)(0,0 ; z) \leq\|G\|^{2}, \quad\|G(z)\| \leq\right| \operatorname{Im} z\right|^{-1} .
$$

This and (4.25) lead to the bound

$$
\mathbf{E}\left\{|D(x ; z)|^{2}\right\} \leq \varphi_{0}^{2} S_{R_{G}} / R_{G}^{d}|\operatorname{Im} z|^{4} .
$$

Plugging it in (4.24), we get

$$
\left|\varepsilon_{R_{G}}(z)\right| \leq \varphi_{0} S_{R_{G}}^{1 / 2} / R_{G}^{d / 2}|\operatorname{Im} z|^{2} \leq C / R_{G}^{d / 2}|\operatorname{Im} z|^{2},
$$

where $C$ does not depend on $z$ and $R_{G}$ if $R_{G}$ is sufficiently large, because by (3.3) and (4.15) we have

$$
\lim _{R_{G} \rightarrow \infty} S_{R_{G}}=1
$$

Hence, (4.12) and (4.29) imply

$$
\left|f_{R_{G}}(z)-f^{(0)}\left(z+w^{2} f_{R_{G}}(z)\right)\right| \leq C\left(R_{G}^{-d / 2}|\operatorname{Im} z|^{-2}+\left|S_{R_{G}}^{1 / 2}-1 \| \operatorname{Im} z\right|^{-3}\right) .
$$

From (4.21) and the bound $|G(0,0 ; z)| \leq\|G(z)\| \leq|\operatorname{Im} z|$ (see (4.28)), it follows that the functions $\left\{f_{R_{G}}\right\}$ are analytic for $\operatorname{Im} z \neq 0$ and are uniformly bounded in $R_{G}$ : $\left|f_{R_{G}}\right| \leq$ $|\operatorname{Im} z|^{-1}$. Thus, restricting $z$ to a compact set $K \subset \mathbb{C} \backslash \mathbb{R}$, we can find a subsequence $\left\{f_{R_{G}^{(l)}}\right\}_{l}$ that converges to a certain limit $\widehat{f}$ uniformly in $K$ and satisfies (2.11). Moreover, since $\operatorname{Im} f_{R_{G}}(z) \operatorname{Im} z>0, \operatorname{Im} z \neq 0$ (see (4.21)), we have $\operatorname{Im} \widehat{f}(z) \operatorname{Im} z \geq 0, \operatorname{Im} z \neq 0$. Recalling that (2.11) is uniquely solvable in this class, we conclude that $\widehat{f}=f$.

The proofs of the claims for $a=d, n_{W}$, and $R_{W}$ essentially follow that for $a=R_{G}$, although they are more involved technically.

(ii) The case where $a=n_{V}$. Again, it suffices to prove the convergence of the Stieltjes transform

$$
f_{n_{V}}(z)=n^{1} \sum_{\alpha=1}^{n} \mathbf{E}\{G(0, \alpha ; 0, \beta ; z)\}
$$

(see (3.17)-(3.18) ) of $N_{n_{V}}$ to a solution of (2.23). In essence, we follow the proof of Theorem 2.3 in 39 .

We begin with proving a counterpart of Lemma 4.2. For this, we use an analog of the differential formula (4.18). Suppose $\Phi: U(n) \rightarrow \mathbb{C}$ admits a $C^{1}$ continuation to an open neighborhood of $U(n)$ in the entire algebra of $(n \times n)$ matrices with complex entries. Denote by $\mathbf{E}_{U}\{\ldots\}$ integration with respect to the Haar measure on $U(n)$ normalized to unity. Then for any Hermitian $X$ we have

$$
\mathbf{E}_{U}\left\{\Phi^{\prime}(U) \cdot U X\right\}=\mathbf{E}_{U}\left\{\Phi^{\prime}(U) \cdot X U\right\}=0,
$$

where $\Phi^{\prime}$ is viewed as a linear map from $\mathcal{M}_{n}(\mathbb{C})$ to $\mathbb{C}$. 
To prove (4.33), it suffices to use the fact that the Haar measure is invariant with respect to the left and the right group shift. Taking $e^{i \varepsilon X}$ as the shift matrix and differentiating the $\varepsilon$-independent quantities $\mathbf{E}\left\{\Phi\left(U e^{i \varepsilon X}\right)\right\}$ and $\mathbf{E}\left\{\Phi\left(e^{i \varepsilon X} U\right)\right\}$ with respect to $\varepsilon$ at $\varepsilon=0$, we obtain (4.33).

By using (4.33) with $U=U(x)$ and $\Phi=G_{n_{V}}(x, \gamma ; y, \beta ; z)$, where

$$
G_{n_{V}}(z)=\left(H_{n_{V}}-z\right)^{-1}=\left\{G_{n_{V}}(x, \alpha ; y, \beta ; z)\right\}_{x, y \in \mathbb{Z}^{d}, \alpha, \beta=1, \ldots, n_{V}},
$$

and employing an analog of (4.20), we see that, for any $\gamma, \beta=1, \ldots, n_{V}$ and $x, y \in \mathbb{Z}^{d}$, we have

$$
\mathbf{E}\left\{\left(G_{n_{V}}(x, x ; z)\left[X, U^{*}(x) B U(x)\right] G_{n}(x, y ; z)\right)_{\gamma \beta}\right\}=0,
$$

where $G_{n}(x, y ; z)=\left\{G_{n}(x, \alpha ; y, \beta ; z)\right\}_{\alpha, \beta=1}^{n}$ is an $(n \times n)$ matrix, $[A, B]=A B-B A$, and here and below we write $n$ instead of $n_{V}$. Then we choose $X=\left\{\delta_{\alpha \alpha^{\prime}} \delta_{\gamma \gamma^{\prime}}\right\}_{\alpha^{\prime}, \gamma^{\prime}=1}^{n}$ in (4.33) to obtain the relation

$$
\begin{aligned}
& \mathbf{E}\left\{G_{n}(x, \gamma ; x, \gamma ; z)\left(U^{*}(x) B U(x) G_{n}(z)\right)(x, \alpha ; y, \beta)\right\} \\
& \quad=\mathbf{E}\left\{\left(G_{n}(x, x ; z) U^{*}(x) B U(x)\right)_{\gamma \gamma} G_{n}(x, \alpha ; y, \beta ; z)\right\} .
\end{aligned}
$$

Applying the operation $n^{-1} \sum_{\gamma=1}^{n}$ to it, we arrive at the matrix identity

$$
\mathbf{E}\left\{\delta_{n, B}(x) G_{n}(x, y ; z)\right\}=\mathbf{E}\left\{g_{n}(x ; z) U^{*}(x) B U(x) G_{n}(x, y ; z)\right\},
$$

where

$$
\begin{aligned}
\delta_{n, B}(x ; z) & =n^{-1} \operatorname{tr} G_{n}(x, x ; z) U^{*}(x) B U(x), \\
g_{n}(x ; z) & =n^{-1} \operatorname{tr} G_{n}(x, x ; z),
\end{aligned}
$$

and "tr" denotes the sum of the diagonal entries of the corresponding $(n \times n)$ matrix. Introducing the centered quantities

$$
g_{n}^{\circ}(x ; z)=g_{n}(x ; z)-\mathbf{E}\left\{g_{n}(x ; z)\right\}, \delta_{n, B}^{\circ}(x, z)=\delta_{n, B}(x ; z)-\mathbf{E}\left\{\delta_{n, B}(x ; z)\right\}
$$

and observing that, since $\{U(x)\}_{x \in \mathbb{Z}^{d}}$ are independent and identically distributed unitary matrices and the first term on the right in (3.14) depends only on $(x-y)$, i.e., the operator $H_{n V}$ in (3.14) satisfies (3.15), for all $x \in \mathbb{Z}^{d}$ we have

$$
\begin{aligned}
\mathbf{E}\left\{g_{n}(x ; z)\right\} & =\mathbf{E}\left\{g_{n}(z)\right\}, \quad g_{n}(z)=g_{n}(0 ; z), \\
\mathbf{E}\left\{\delta_{n, B}(x ; z)\right\} & =\mathbf{E}\left\{\delta_{n, B}(z)\right\}, \quad \delta_{n, B}(z)=\delta_{n, B}(0 ; z) .
\end{aligned}
$$

Thus, writing the relation

$$
U^{*}(x) B U(x) G_{n}(x, y)=\delta(x-y)-\left(\left(H^{(0)}-z\right) G_{n}\right)(x, y),
$$

which follows from the resolvent identity for the pair $\left(H_{n}, H^{(0)}\right)$ with

$$
H^{(0)}=\left\{h(x-y) \delta_{a \beta}\right\}_{x, y \in \mathbb{Z}^{d}, \alpha, \beta=1, \ldots, n},
$$

we obtain

$$
\begin{aligned}
\mathbf{E}\left\{\delta_{n, B}\right\} & \mathbf{E}\left\{G_{n}(x, y)\right\}+\mathbf{E}\left\{\delta_{n, B}^{\circ}(x ; z) G_{n}(x, y)\right\} \\
= & \mathbf{E}\left\{g_{n}\right\}\left(\delta(x-y)+z \mathbf{E}\left\{G_{n}(x, y)\right\}-\mathbf{E}\left\{\left(H^{(0)} G_{n}\right)(x, y)\right\}\right) \\
& +\mathbf{E}\left\{g_{n}^{\circ}(x ; z) U^{*}(x) B U(x) G_{n}(x, y)\right\},
\end{aligned}
$$

or, after regrouping terms,

$$
\begin{aligned}
& f_{n}(z)\left(\left(H^{(0)}-h_{n, B}(z)\right) \mathbf{E}\left\{G_{n}\right\}\right)(x, y) \\
& \quad=f_{n}(z) \delta(x-y)+\mathbf{E}\left\{g_{n}^{\circ}(x ; z) U^{*}(x) B U(x) G_{n}(x, y)\right\}-\mathbf{E}\left\{\delta_{n, B}^{\circ}(x ; z) G_{n}(x, y)\right\}
\end{aligned}
$$


where

$$
f_{n}(z)=\mathbf{E}\left\{g_{n}(z)\right\}, \quad h_{n, B}(z)=z-\mathbf{E}\left\{\delta_{n, B}(z)\right\} f_{n}^{-1} .
$$

Since the measure $N^{(0)}$ in (4.3) has compact support (see (3.2)) and $B$ satisfies (4.8), the functions $f_{n}$ and $h_{n, B}$ have the same properties as those in the proof of Theorem 2.3 in 39. In particular, for any $z$ in the domain

$$
\Gamma_{\alpha, \beta}=\{z \in \mathbb{C}:|\operatorname{Re} z| \leq \alpha|\operatorname{Im} z|,|\operatorname{Im} z| \geq \beta\}, \quad \beta \geq(\alpha+1) m_{B}^{(1)},
$$

we have the bound

$$
\left|\operatorname{Im} h_{n, B}(z)\right| \geq \beta \frac{\beta-(\alpha+3) M}{\beta-2 M} .
$$

Hence, the operator $H^{(0)}-h_{n, B}(z)$ in (4.36) is invertible:

$$
G^{(0)}\left(h_{n, B}(z)\right)=\left(H^{(0)}-h_{n, B}(z)\right)^{-1}, \quad z \in \Gamma_{\alpha, \beta},
$$

and

$$
\left\|G^{(0)}\left(h_{n, B}(z)\right)\right\| \leq\left(\beta \frac{\beta-(\alpha+3) M}{\beta-2 M}\right)^{-1}, \quad z \in \Gamma_{\alpha, \beta} .
$$

Now, multiplying (4.36) by $G^{(0)}\left(h_{n, B}(z)\right)$ from the left and applying the operation $n^{-1} \mathrm{tr}$ to the result, we obtain

$$
f_{n}(z) \mathbf{E}\left\{n^{-1} \operatorname{tr} G_{n}(x, y)\right\}=f_{n} G^{(0)}\left(x-y ; h_{n, B}(z)\right)+\varepsilon_{n}(x, y),
$$

where

$$
\begin{aligned}
\varepsilon_{n}(x, y)=\mathbf{E}\left\{g_{n}^{\circ}(x ; z) n^{-1} \operatorname{tr}\left(G^{(0)}\left(h_{n, B}\right) H^{(1)} G_{n}\right)(x, y)\right\} \\
-\mathbf{E}\left\{\delta_{n, B}^{\circ}(x ; z) n^{-1} \operatorname{tr}\left(G^{(0)}\left(h_{n, B}\right) G_{n}\right)(x, y)\right\}
\end{aligned}
$$

and

$$
H^{(1)}(x, y)=U^{*}(x) B U(x) \delta(x-y) .
$$

Next, the resolvent identities for $\left(H_{n}, H^{(0)}\right)$ and $\left(H^{(0)}, 0\right)$ imply that

$$
G^{(0)}\left(h_{n, B}\right) H^{(1)} G_{n}=\left(z-h_{n, B}\right) G^{(0)}\left(h_{n, B}\right) G_{n}+G^{(0)}\left(h_{n, B}\right)-G_{n},
$$

and for $z \in \Gamma_{\alpha, \beta}$ we have

$$
\left\|G^{(0)}\left(h_{n, B}\right) H_{1} G\right\| \leq \frac{\left|z-h_{n, B}\right|(\beta-2 M)}{(\beta-(\alpha+3) M) \beta^{2}}+\frac{\beta-2 M}{\beta-(\alpha+3) M}+\frac{1}{\beta} \leq \frac{\left|z-h_{n, B}\right|+C}{(\beta-(\alpha+3) M) \beta} .
$$

Thus, for the remainder matrix $\varepsilon_{n}$ in (4.42) we obtain

$$
\left\|\varepsilon_{n}(x, y)\right\| \leq \frac{\left(\left|z-h_{n, B}\right|+C\right) \operatorname{Var}^{1 / 2}\left\{g_{n}\right\}+\operatorname{Var}^{1 / 2}\left\{\delta_{B}\right\}}{(\beta-(\alpha+3) M)} .
$$

Now we need a bound for the variance of a function of $\{U(x)\}_{x \in \mathbb{Z}^{d}}$, analogous to the Poincaré-Nash type inequality (4.26). In 39] it was shown that if $U$ is the random Haar distributed unitary matrix and $\Phi: U(n) \rightarrow \mathbb{C}$ admits a $C^{1}$ extension to an open neighborhood of $U(n)$ in the algebra of complex $(n \times n)$-matrices, then

$$
\operatorname{Var}\{\Phi\} \leq \frac{1}{n} \mathbf{E}\left\{\sum_{\alpha, \beta=1}^{n}\left|\Phi_{\alpha \beta}^{\prime}(x)\right|^{2}\right\}
$$

where

$$
\Phi_{j k}^{\prime}=\lim _{\varepsilon \rightarrow 0}\left(\Phi\left(U+\varepsilon E_{j k}\right)-\Phi(U)\right) \varepsilon^{-1}
$$

and $E_{j k}$ is the $(n \times n)$-matrix whose entries are zero except the $j k$ th entry equal to 1 . 
By using a standard martingale type argument, we conclude that if $\Omega$ is the probability space formed by the product over $x \in \mathbb{Z}^{d}$ of groups $U_{x}(n)$ with their Haar measures, $\mathbf{E}\{\ldots\}$ is the corresponding expectation, and $\Phi: \Omega \rightarrow \mathbb{C}$ admits a $C^{1}$ extension with respect to every argument $U(x) \in U_{x}(n), x \in \mathbb{Z}^{d}$, to an open neighborhood of $U_{x}(n)$ in the algebra of complex $(n \times n)$-matrices, then

$$
\operatorname{Var}\{\Phi\} \leq \frac{1}{n} \mathbf{E}\left\{\sum_{x \in \mathbb{Z}^{d}} \sum_{\alpha, \beta=1}^{n}\left|\Phi_{\alpha \beta}^{\prime}(x)\right|^{2}\right\},
$$

where

$$
\Phi_{\alpha \beta}^{\prime}(x)=\lim _{\varepsilon \rightarrow 0}\left(\Phi\left(\ldots, U_{n}(x)+\varepsilon E_{\alpha \beta}, \ldots\right)-\Phi\left(\ldots, U_{n}(x), \ldots\right)\right) \varepsilon^{-1} .
$$

Applying this bound to $g_{n}(z)$, we see that

$$
\begin{aligned}
\operatorname{Var}\left\{g_{n}(z)\right\} \leq \frac{1}{n^{3}} \sum_{x \in \mathbb{Z}^{d}} \sum_{\alpha, \beta=1}^{n} \mathbf{E}\{ & \mid(B U(x) G(x, 0) G(0, x))_{\alpha \beta} \\
& \left.\quad-\left.\left(G(x, 0) G(0, x) U^{*}(x) B\right)_{\beta \alpha}\right|^{2}\right\} \\
\leq & \frac{4 m_{B}^{(2)}}{n^{2}|\operatorname{Im} z|^{4}},
\end{aligned}
$$

and similarly

$$
\operatorname{Var}\left\{\delta_{n, B}(x ; z)\right\} \leq \frac{8 m_{B_{n}}^{(4)}}{n^{2}|\operatorname{Im} z|^{4}}
$$

where

$$
m_{B_{n}}^{(l)}=\int_{\mathbb{R}}|\lambda|^{l} N_{B_{n}}(d \lambda),
$$

and we have used the bounds $\|G(x, y)\| \leq|\operatorname{Im} z|^{-1}$ ( $\operatorname{cf}(4.28)$ ) and $\left|\operatorname{tr} B^{2} A\right| \leq\|A\| \operatorname{tr} B^{2}$, valid for any Hermitian $B$ and an arbitrary $A$. Thus, uniformly in $z$ varying on a compact set $K \subset \Gamma_{\alpha, \beta}$, we have

$$
\left\|\varepsilon_{n}(x, y)\right\|=O\left(n^{-1}\right) .
$$

Setting $x=y=0$ in (4.41) and using (4.37), we obtain

$$
f_{n}(z)=f^{(0)}\left(h_{n, B}(z)\right)+\varepsilon_{n}(0,0) f_{n}^{-1}(z),
$$

where

$$
f^{(0)}(z)=G^{(0)}(0,0 ; z)=\int \frac{N^{(0)}(d \lambda)}{\lambda-z} .
$$

Now a standard compactness argument (see the end of the proof of assertion (i)) leads to the first equation of the system (2.23) in the limit as $n \rightarrow \infty$.

The second equation can be obtained by interchanging $H^{(0)}$ and $B$, i.e., by the same procedure for the operator defined by the matrix

$$
\left\{\left(U(x) h(x-y) U^{*}(y)\right)_{\alpha, \beta}+B_{\alpha, \beta} \delta(x-y)\right\}
$$

and having the same spectrum as (3.14).

The third equation follows from the resolvent identity for the pair $\left(H_{n}, 0\right)$. 


\section{$\S 5$. Discussion}

As was mentioned above, the operators $H_{a}$ with $a=R_{G}, d, R_{W}, n_{W}, n_{V}$ are analogs of certain Hamiltonians of lattice models of statistical mechanics, where the limits of infinite interaction radius, dimension, or the number of spin components lead to the mean field or the spherical versions of the models. On the other hand, the studies of elementary excitations and wave propagation in disordered media are based substantially on the spectral properties of the Schrödinger operator with random potential and its discrete analog (known also as the Anderson model), the sum of the finite difference Laplacian $-\Delta$ and the multiplication operator $V$ determined by the collection of independent and identically distributed random variables $\{V(x)\}_{x \in \mathbb{Z}^{d}}$ :

$$
-\Delta+V
$$

Spectral analysis of this operator and other finite difference and differential operators with random ergodic coefficients is among the main objectives of the ROT and of the branch of condensed matter theory known as the theory of disordered systems. In particular, the theory includes several approximation schemes, similar to the mean field approximations in statistical mechanics (see, e.g., [24, Chapter 5]). One may ask then about the meaning of the results of this section in the context of the ROT and the theory of disordered systems. It can be shown that the result mentioned in Theorem 4.1 for $a=n_{W}$ with $\widehat{\varphi}=a \mathbf{1}_{A}, a>0, A \subset \mathbb{R}^{d}$, i.e., with $\sigma$ in (2.20) having atoms at zero and $a$, the latter with the mass meas $A$, corresponds to the so-called modified propagator approximation, and the result of Theorem 4.1 (v) corresponds to the so-called coherent potential approximation. We refer the reader to [24, Chapter 5], and to [22, 31, 40, 50] for related discussion and references.

Note that the above approximations are widely used to describe the transport properties of disordered systems that involve a sufficiently large amount of eigenfunctions nondecaying at infinity (delocalized or extended states in physics), usually associated with the generalized eigenfunctions of the absolutely continuous spectrum of the corresponding random ergodic operators. On the other hand, the point component of the spectrum associated with eigenfunctions decaying (as a rule exponentially) at infinity (localized states in physics) does not contribute to the transport, despite that the point spectrum is dense as a rule (see, e.g., 24, 23] for explanations).

The point spectrum of the Schrödinger operator with random weakly correlated potential is fairly well understood by now. In the one-dimensional case $(d=1)$ the spectrum is pure point and the corresponding eigenfunctions decay exponentially at infinity (see, e.g., [12, 36] and the references therein), and this is also the case for any dimension for certain neighborhoods of spectrum edges or if the potential is sufficiently large (see, e.g., [1, 10] for recent results and references).

The situation is much less satisfactory for the absolutely continuous component. It is widely believed and supported by many physical and numerical results that if $d \geq 3$ and the random potential is sufficiently small, then the absolutely continuous component is not empty and lies sufficiently far from the spectrum edges. Moreover, the pure point and absolutely continuous components do not overlap, so that they can only meet at points, which are known in physics literature as the mobility edges, because if at zero temperature the Fermi level of the electron gas moves from the absolutely continuous spectrum to the pure point one, then the electron conductivity vanishes. This is called the Anderson metal-insulator transition. It is a fundamental problem to establish the existence of the absolutely continuous spectrum of random ergodic operators, the transition, and their properties (see, e.g., 43] for a recent discussion of related topics). 
Due to the mean field character of random matrices, demonstrated by Theorem 4.1, they are simpler to study and are always in the delocalized regime (see, e.g., Subsection 2.2.1 (ii)). In view of this and the theorem, it is tempting to believe that the location of the absolutely continuous spectrum of the operators $H_{a}$ with sufficiently large $a$ might be close to the support of the large size limit $N$ of the normalized counting measure (2.10) of eigenvalues of random matrices, described in the theorem and 92 , and that the pure point spectrum is in $\mathbb{R} \backslash \operatorname{supp} N$.

Here are several facts supporting this belief.

1) It is widely believed and proved in many cases that, for the Lifshitz tails of the IDS of ergodic operators, the asymptotics of $N_{a}$ (exponentially decaying near the spectrum edges, see, e.g., [12] and [36, Chapter IV]), or more generally, a sufficiently small IDS, is intimately related to the pure point spectrum of ergodic operators. Theorem 4.1 and the results described in $\oint_{2}$, in particular, formulas (2.14) and (2.21), (2.22) show that the Lifshitz tails of $N_{a}$ vanish as $a \rightarrow \infty$. Moreover, the spectral characteristics of random operators (as well as physical characteristics of the dynamics of elementary excitations in disordered media) are absolutely continuous with respect to the IDS. Recall that, by Theorem 4.1 for the operators $H_{a}$ of Subsection 3.1, we have

$$
\lim _{a \rightarrow \infty} N_{a}(\Delta)=0, \quad \Delta \subset \mathbb{R} \backslash \operatorname{supp} N .
$$

Hence, according to the belief, the pure point spectrum of $H_{a}$ in the set $\mathbb{R} \backslash \operatorname{supp} N$ must be suppressed if $a$ is sufficiently large, and the contribution of this part of the spectrum to physical characteristics must vanish as $a \rightarrow \infty$ (see, e.g., 22 for the corresponding results on the electric conductivity).

2 ) In this item, for simplicity we discuss the case where $a=d$; however the conclusions can be applied to all other values of $a$ with obvious modifications.

Recall that if $m$ is a finite measure with atoms $\left\{m_{j}\right\}$, and $\widehat{m}$ is its Fourier transform, then by the Wiener theorem we have

$$
\lim _{T \rightarrow \infty} \int_{0}^{T}|\widehat{m}(t)|^{2} d t=\sum_{j}\left|m_{j}\right|^{2} .
$$

The Stieltjes transform version of that theorem applied to the Green function $G_{d}$ of (3.6) - 3.9), written via the spectral theorem, yields

$$
\lim _{\varepsilon \rightarrow 0} \frac{\varepsilon}{\pi} \int_{\Delta} \mathbf{E}\left\{\left|G_{d}(x, 0: \lambda+i \varepsilon)\right|^{2}\right\} d \lambda=\mathbf{E}\left\{\sum_{\lambda_{l} \in \Delta \cap \sigma_{p p}\left(H_{d}\right)}\left|\psi_{l}(x) \psi_{l}(0)\right|^{2}\right\},
$$

where $\sigma_{p p}$ is the point spectrum of $H_{d}$, the $\left\{\lambda_{l}\right\}$ are its eigenvalues, the $\psi_{l}$ are the corresponding eigenfunctions, and we assume that the point spectrum is simple. Summing this over $x \in \mathbb{Z}^{d}$, on the right-hand side we obtain

$$
N_{d}^{(l o c)}(\Delta):=\mathbf{E}\left\{\sum_{\lambda_{l} \in \Delta \cap \sigma_{p p}\left(H_{d}\right)}\left|\psi_{l}(0)\right|^{2}\right\} .
$$

Comparing this with formula (3.16) for $a=d$, written symbolically via the spectral theorem as

$$
N_{d}(\Delta)=\mathbf{E}\left\{\int_{\Delta \cap \sigma_{c}\left(H_{d}\right)}\left|\psi_{\lambda}(0)\right|^{2} n(d \lambda)\right\}+\mathbf{E}\left\{\sum_{\lambda_{l} \in \Delta \cap \sigma_{p p}\left(H_{d}\right)}\left|\psi_{l}(0)\right|^{2}\right\},
$$

(see, e.g., 7] for the precise form), where $\sigma_{c}$ is the continuous spectrum of $H_{d}$, we conclude that

$$
N_{d}^{(l o c)}(\Delta) \leq N_{d}(\Delta)
$$


It is known [36] that $\Delta \subset \mathbb{R}$ contains the spectrum of $H_{d}$ with probability 1 if and only if $N_{d}(\Delta)>0$. Similarly, it can be shown that $\Delta$ contains the point spectrum of $H_{d}$ with probability 1 if and only if $N_{d}^{(l o c)}(\Delta)>0$, the same is true for the continuous spectrum, and $N_{d}^{(c)}:=N_{d}-N_{d}^{(\mathrm{loc})}$. Thus, $N_{d}^{(\mathrm{loc})}$ can be called the integrated density of the point spectrum (localized states) and $N_{d}^{(c)} \geq 0$ can be called the integrated density of the continuous spectrum.

Now, assume that, in the spirit of Theorem 4.1 for $N_{d}^{(\text {loc })}$ of (5.2)-(5.3) we have

$$
\lim _{d \rightarrow \infty} N_{d}^{(l o c)}(\Delta)=0
$$

for some (or even for all) $\Delta \subset \operatorname{supp} N$, where $N=\lim _{d \rightarrow \infty} N_{d}$ is defined by (2.11) . Then $N_{d}^{(c)}(\Delta)>0$ for all sufficiently large $d$, so that $\Delta$ contains the continuous spectrum of $H_{d}$ for the same $d$.

The above could be viewed as a simple looking (but not necessarily simply implementable) scenario to prove the existence of the continuous spectrum of $H_{d}$ for sufficiently large $d$ (more generally, sufficiently large $a$ ).

3) We mention one more widely believed property that "feels" the difference between the (pure) point and (absolutely) continuous spectrum of random ergodic operators. This property was first formulated and actively studied in the RMT, then turned out to be of interest in the study of quantum chaos, and then in the ROT. In the RMT it is known as the local eigenvalue statistics and deals with the behavior of eigenvalues of large random matrices on the intervals of the spectral axis whose length is of the order of the mean distance between eigenvalues. A basic quantity here is the gap probability defined in (2.15).

In the case of ergodic operators, we need to consider their finite box restrictions (see $H_{a \Lambda}$ in Subsection 3.3). For operators with weakly correlated ergodic coefficients and with $\lambda_{0}$ of (2.15) lying in the pure point spectrum, the local eigenvalue statistics is Poisson, i.e., coincides with that for independent points. In particular, the righthand side of (2.16) is $e^{-s}$ (see, e.g., [10] for recent rigorous results and references). On the other hand, it is widely believed and supported by numerous arguments in the physics literature (see [13]) that the local eigenvalue statistics in the absolutely continuous spectrum coincides with that for the Gaussian random matrices. In particular, for the Schrödinger operator with random potential and for its discrete counterpart (5.1) we expect certain analogs of formulas (2.16)-(2.18) for the real symmetric version of (2.1), (2.2) known as the Gaussian orthogonal ensemble (GOE) (see [27, Chapter 7], while the GUE formulas (2.16)-(2.18) are expected for the Schrödinger operator with random potential and constant magnetic field (where the time reversal symmetry is absent). The recent papers [4, 48, provide a rigorous support for this belief.

4) An interesting and natural intermediate case between the Gaussian (more generally, Wigner) matrices and random ergodic operators is given by the band matrices (2.3). They can be viewed as a finite box version of $H_{R_{G}}$ in which the size of the box and $R_{G}$ tend to infinity simultaneously, see, e.g., 43 for a recent review. It is conjectured and supported by recent rigorous results [41] that if $b_{n} / n^{1 / 2} \rightarrow 0$ in (2.3) as $n \rightarrow \infty$, then the eigenvectors are localized in the sense that they are essentially supported on an $O(1)$ part of $[1, n]$ as $n \rightarrow \infty$, and if $b_{n} / n^{1 / 2} \rightarrow \infty$, then the eigenvectors are delocalized, as in the case of the Gaussian (see item (iii) of Subsection 2.2.1) and the Wigner matrices [14. For rigorous supports of these expectations, see [14, 15. As for the border case of $b_{n}$ proportional to $n^{1 / 2}$, one expects a kind of transition between the localized and delocalized states. This transition was studied in the physics paper [28] and a review of state of the art and rigorous results were given in [43]. 


\section{RefERENCES}

[1] M. Aizenman, F. Germinet, A. Klein, and S. Warzel, On Bernoulli decompositions for random variables, concentration bounds, and spectral localization, Probab. Theory Related Fields 143 (2009), 219-238. MR2449128(2009k:60042)

[2] N. I. Akhiezer and I. M. Glazman, Theory of linear operators in Hilbert space. Vols. 1, 2, 3rd ed., Vishcha Shkola, Khar'kov, 1977, 1978; English transl., Dover, New York, 1993. MR0486990 (82g:47001a) MR0509335(82g:47001b) MR.1255973 (94i:47001)

[3] G. W. Anderson, A. Guionnet, and O. Zeitouni, An introduction to random matrices, Cambridge Stud. in Adv. Math., vol. 118, Cambridge Univ. Press, Cambridge, 2010. MR2760897

[4] S. Bachmann and W. De Roeck, From the Anderson model on a strip to the DMPK equation and random matrix theory, arXiv:0912.1574.

[5] Z. Bai and J. W. Silverstein, Spectral analysis of large dimensional random matrices, Springer, New York, 2010. MR 2567175 (2011d:60014)

[6] G. Ben Arous and S. Péché, Universality of local eigenvalue statistics for some sample covariance matrices, Comm. Pure Appl. Math. 58 (2005), 1316-1357. MR2162782 (2006h:62072)

[7] Yu. M. Berezanskiŭ, Expansions in eigenfunctions of selfadjoint operators, Naukova Dumka, Kiev, 1965; English transl., Transl. Math. Monogr., vol. 17, Amer. Math. Soc., Providence, RI, 1968. MR0222719 (36:5769); MR0222718(36:5768)

[8] V. Bogachev, Gaussian measures, Nauka, Moscow, 1997; English transl., Math. Surveys Monogr., vol. 62, Amer. Math. Soc., Providence, RI, 1998. MR 1663929 (2000b:60097) MR1642391 (2000a:60004)

[9] E. Brézin and S. Hikami, Correlations of nearby levels induced by a random potential, Nuclear Phys. B 479 (1996), 697-706. MR1418841(97j:82080)

[10] J.-M. Combes, F. Germinet, and A. Klein, Generalized eigenvalue-counting estimates for the Anderson model, J. Stat. Phys. 135 (2009), 201-216. MR2505733 (2010j:82053)

[11] M. Disertori, H. Pinson, and T. Spencer, Density of states for random band matrices, Comm. Math. Phys. 232 (2002), 83-124. MR.1942858(2004c:82052)

[12] M. Disertori, W. Kirsch, A. Klein, F. Klopp, and V. Rivasseau, Random Schrödinger operators, Panoramas et Synthèses [Panoramas and Syntheses], vol. 25, Soc. Math. France, Paris, 2008. MR.2516524 (2010a:82030)

[13] K. Efetov, Supersymmetry in disorder and chaos, Cambridge Univ. Press, Cambridge, 1997. MR 1628498 (99m:82001)

[14] L. Erdös, Universality of Wigner random matrices: a survey of recent results, arXiv:1004.0801.

[15] L. Erdös and A. Knowles, Quantum diffusion and eigenfunction delocalization in a random band matrix model, arXiv:1002.1695. MR2782623

[16] P. Forrester, Log-gas and random matrices, available at http://www.ms. unimelb.edu.au/ matpjf/matpjf.html

[17] Y. V. Fyodorov and A. D. Mirlin, Scaling properties of localization in random band matrices: a $\sigma$-model approach, Phys. Rev. Lett. 67 (1991), 2405-2409. MR1130103 (92g:82048)

[18] P. C. Hemmer and J. L. Lebowitz, Systems with weak long-range potentials, Phase Transitions and Critical Phenomena. Vol. 5b (C. Domb and M. S. Green, eds.), Acad. Press, London, 1976, pp. 107-203. MR0503012 (58:19880)

[19] K. Johansson, Universality of the local spacing distribution in certain ensembles of Hermitian Wigner matrices, Comm. Math. Phys. 215 (2001), 683-705. MR1810949 (2002j:15024)

[20] _ Universality for certain Hermitian Wigner matrices under weak moment conditions, arXiv:0910.4467.

[21] A. Khorunzhy, B. Khoruzhenko, L. Pastur, and M. Shcherbina, Large- $n$ limit in statistical mechanics and the spectral theory of disordered systems, Phase Transitions and Critical Phenomena. Vol. 15 (C. Domb, J. Lebowitz, eds.), Acad. Press, New York, 1992, pp. 74-239.

[22] A. Khorunzhy and L. Pastur, Limits of infinite interaction radius, dimensionality and the number of components for random operators with off-diagonal randomness, Comm. Math. Phys. 153 (1993), 605-646. MR 1218934 (94g:82024)

[23] W. Kirsch, O. Lenoble, and L. Pastur, On the Mott formula for the ac conductivity and binary correlators in the strong localization regime of disordered systems, J. Phys. A 36 (2003), 1215712180. MR2025824 (2005c:82080)

[24] I. M. Lifshits, S. A. Gredeskul, and L. A. Pastur, Introduction to the theory of disordered systems, Nauka, Moscow, 1982; English transl., Wiley, New York, 1988. MR0672422 (84j:82084) MR1042095 (90k:82073)

[25] A. Lytova and L. Pastur, Central limit theorem for linear eigenvalue statistics of random matrices with independent entries, Ann. Probab. 37 (2009), 1778-1840. MR2561434(2011c:60024) 
[26] V. A. Marchenko and L. A. Pastur, The eigenvalue distribution in some ensembles of random matrices, Mat. Sb. 72 (114) (1967), no. 4, 507-536; English transl., Math. USSR-Sb. 1 (1967), 457-483. MR0208649(34:8458)

[27] M. L. Mehta, Random matrices, Pure Appl. Math., vol. 142, Elsevier, Amsterdam, 2004. MR:2129906 (2006b:82001)

[28] A. Mirlin and Ya. Fyodorov, The statistics of eigenvector components of random band matrices: analytical results, J. Phys. A 26 (1993), L551-L558. MR1236144 (94f:82036)

[29] S. A. Molchanov, L. A. Pastur, and A. M. Khorunzhǐ, Distribution of the eigenvalues of random band matrices in the limit of their infinite order, Teoret. Mat. Fiz. 90 (1992), no. 2, 163-178; English transl., Theoret. and Math. Phys. 90 (1992), 108-118. MR.1182292 (93g:82056)

[30] R. J. Muirhead, Aspects of multivariate statistical theory, Wiley, New York, 1982. MR0652932 (84c:62073)

[31] P. Neu and R. Speicher, Rigorous mean-field model for coherent-potential approximation: Anderson model with free random variables, J. Stat. Phys. 80 (1995), 1279-1308. MR.1349982 (96i:82102)

[32] A. Pajor and L. Pastur, On the limiting empirical measure of eigenvalues of the sum of rank one matrices with log-concave distribution, Studia Math. 195 (2009), 11-29. MR2539559(2010h:15022)

[33] L. Pastur, The spectrum of random matrices, Teoret. Mat. Fiz. 10 (1972), no. 1, 102-112; English transl., Theoret. and Math. Phys. 10 (1972), 67-74. MR0475502 (57:15106)

[34] - A simple approach to the global regime of Gaussian ensembles of random matrices, Ukrain. Mat. Zh. 57 (2005), no. 6, 790-817; English transl., Ukrainian Math. J. 57 (2005), no. 6, 936-966. MR2208456 (2007d:82079)

[35] _ Eigenvalue distribution of random matrices, Random Media 2000 (J. Wehr, ed.), Lectures from the Summer School "Random Media 2000" held in Mandralin, Poland. ICM Warsaw Univ., Warsaw, 2007, pp. 95-206.

[36] L. Pastur and A. Figotin, Spectra of random and almost-periodic operators, Grundlehren Math. Wiss., Bd. 297, Springer, Berlin, 1992. MR1223779 (94h:47068)

[37] L. Pastur and M. Shcherbina, Bulk universality and related properties of Hermitian matrix models, J. Stat. Phys. 130 (2008), 205-250. MR2375744 (2008k:82050)

[38] L. Pastur and V. Vasilchuk, On the law of addition of random matrices, Comm. Math. Phys. 214 (2000), 249-286. MR.1796022 (2002b:60037)

[39] _ On the law of addition of random matrices: covariance and the central limit theorem for traces of resolvent, Probability and Mathematical Physics, CRM Proc. Lecture Notes, vol. 42, Amer. Math. Soc., Providence, RI, 2007, pp. 399-416. MR2352281(2008k:60054)

[40] D. A. Rowlands, Short-range correlations in disordered systems: nonlocal coherent-potential approximation, Rep. Prog. Phys. 72 (2009), 086501.

[41] J. Schenker, Eigenvector localization for random band matrices with power law band width, Comm. Math. Phys. 290 (2009), 1065-1097. MR2525652 (2010i:60024)

[42] T. Shcherbina, On universality of bulk local regime of the deformed Gaussian unitary ensemble, $\mathrm{Zh}$. Mat. Fiz. Anal. Geom. 5 (2009), 396-433. (English) MR2590774 (2011f:60011)

[43] T. Spencer, Random banded and sparse matrices, The Oxford Handbook of Random Matrix Theory (G. Akemann, J. Baik, Ph. DiFrancesco, eds.), Oxford Univ. Press, Oxford, 2011.

[44] H. Stanley, Introduction to phase transitions and critical phenomena, Clarendon Press, Oxford, 1971.

[45] T. Tao and V. Vu, Random matrices: universality of local eigenvalue statistics, arXiv:0906.0510; Acta Math. 206 (2011), no. 1, 127-204. MR2784665

[46] Random covariance matrices: universality of local statistics of eigenvalues, arXiv: 0912.0966.

[47] C. J. Thompson, Mathematical statistical mechanics, Princeton Univ. Press, Princeton, 1979. MR.0548873 (80h:82001)

[48] V. Valko and B. Virag, Random Schrödinger operators on long boxes, noise explosion and the GOE, arXiv:0912.0097.

[49] D. Voiculescu, Aspects of free probability, XIVth International Congress on Mathematical Physics, World Sci. Publ., Hackensack, NJ, 2005, pp. 145-157. MR2227827 (2007b:46120)

[50] F. Wegner, Disordered electronic systems as a model of interacting matrices, Phys. Rep. 67 (1980), 15-24. MR0600875

Mathematics Division, Institute for Low Temperatures, Kharkov, Ukraine

E-mail address: pastur2001@yahoo.com

Received 15/SEP/2010 